\title{
Review of: "Myalgic encephalomyelitis/chronic fatigue syndrome as a breakdown of homeostasis"
}

\author{
Suzanne Broadbent ${ }^{1}$ \\ 1 University of the Sunshine Coast
}

Potential competing interests: The author(s) declared that no potential competing interests exist.

This article, which reports on ME/CFS as a condition primarily characterised by a breakdown in homeostasis, is an interesting and useful read for patients and clinicians, and sums up some of the more recent evidence. The author has focused on the actions and interactions of the nervous, endocrine and immune systems, and how these may be affected in ME/CFS. Although ME/CFS has been described using text from the CDC, this text is not really a clinical definition as such, and the clinical definition(s) in the IOM Report Guide for Clinicians would really have been a better choice. However, overall the article covers the actions, interactions and known disturbances of the three body systems well. I would have liked to have seen more detail around the immuno-suppressive effects of increased cortisol on some specific immune cells, such as neutrophils and Natural Killer cells, as these cells are frontline, non-specific immune cells which are activated during viral and bacterial challenges. Increased stress, as well as viral infections, will increase cortisol, possibly leading to immuno-suppression and contributing to the development of ME/CFS. The author covers the underlying inflammatory responses found in ME/CFS well, and also the microbiome disturbances and mitochondrial dysnfunction so typical of this condition. There could have been more detail perhaps on how mitochondrial dysfunction and reduced ATP contributes to post-exertional malaise (PEM), which really is the hallmark feature of ME/CFS. Low ATP production and a low anaerobic threshold are real issues for patients with constant or recurring fatigue and who experience difficulties with managing energy levels for daily activities. Overall, the article is a good read and a very useful contribution to the available literature. 